\title{
Remote health care system for elderly people with chronic diseases
}

\author{
Isabel Martí Ruiz \\ $R \& D$ at Tecnologías para la Salud y el Bienestar, Spain \\ imarti@tsbtecnologias.es \\ Juan Pablo Lázaro Ramos \\ $\mathrm{R} \& \mathrm{D}$ at Tecnologías para la Salud y el Bienestar, Spain \\ Alejandro Aracil Ramón \\ $R \& D$ at Tecnologías para la Salud y el Bienestar, Spain
}

\begin{abstract}
Chronic diseases are the leading cause of mortality in the world, representing $60 \%$ of all deaths. The REMOTE project aims to establish a multidisciplinary and integrated approach to utilize information and communication technologies for addressing real needs of elderly people, especially of citizens at risk due to geographic and social isolation in combination with specific chronic conditions. More specifically, REMOTE offers a set of services oriented to maintain the independence of the persons who face chronic diseases and live alone. REMOTE provides four different kinds of services, related to different areas such as environmental home control, schedule control, mental health and physical health. This paper is focussed on the services related to the physical health. An activity advisor, a nutritional advisor and a medical advisor will be analyzed. All services are based on client-server architecture, compound by the server side and two main clients, one for the elderly and another for the doctors who take care of them. Nowadays, the pilot phase of REмоте has just started. Rемоте counts with several pilots, in sites like Norway, Romania, Germany, Israel and Spain in which the elderly participants present different conditions. Also, the pilot sites count with medical doctors specialized in different areas, such as nutrition or physiotherapy. This situation allows testing the services in a wide variety of cases. In this paper we will analyze the results achieved so far in the pilot phase.
\end{abstract}

Keywords: REMOTE, elderly, chronic disease, physical health

\section{Resumen}

Las enfermedades crónicas son la principal causa de mortalidad en el mundo y representan un $60 \%$ de las muertes. El proyecto REMOTE pretende establecer un enfoque multidisciplinario e integrado para utilizar tecnologías de la información y la comunicación para responder a las necesidades reales de las personas de edad avanzada, especialmente de los ciudadanos en situación de riesgo debido al aislamiento geográfico y social en combinación con determinadas enfermedades crónicas. Más específicamente, REMOTE ofrece un conjunto de servicios orientados a mantener la independencia de las personas con enfermedades crónicas que viven en situaciones de aislamiento. RеMOTE brinda un conjunto de servicios relacionados con diferentes áreas tales como el control ambiental del hogar, el control de horarios, la salud mental y la salud física. Este artículo se centra en los servicios relacionados con la salud física. En él se describirán un asesor nutricional, un asesor de ejercicio y un asesor de medicación. Todos los servicios se basan en una arquitectura cliente servidor y se componen de un servidor y dos clientes, uno para los ancianos y otro para los médicos que se encargan de ellos. Actualmente, REMOTE acaba de comenzar su fase de pilotaje. REMOTE cuenta con pilotos en diferentes países, como Noruega, Rumanía, Alemania o España, en los que los participantes presentan 
diferentes condiciones. Además, los pilotos cuentan con médicos especialistas en distintos aspectos, tales como nutricionistas o fisioterapeutas. Esta situación permite analizar el sistema en una gran variedad de casos. En este artículo se describirán los resultados obtenidos hasta la fecha.

Palabras clave: REMOTE, ancianos, enfermedades crónicas, salud física

\section{Introduction}

The world population is aging. This is due to several factors such as increased life expectancy and falling fertility rates, chiefly linked to progress in birth control, the baby boom and migration movements. This tendency is even bigger in developed countries, where there is already a large number of elderly people and this trend is constantly growing. Aging affects the economic and social foundations of societies, since governments need to dedicate more money to take care of the elderly and more support is needed with fewer hands to cater for their needs (EU, 2009).

This situation also presents a challenge for the health care systems, which need to cope with the health problems of the elderly community. This means that the health systems need to be prepared to deal with the so-called "four giants" of geriatrics (Isaacs) as: immobility, instability, incontinence and intellectual impairment. Furthermore, chronic diseases are common in elderly people, such as diabetes, Alzheimer's, cardiovascular diseases, or chronic respiratory diseases.

According to the World Health Organization (wHo, 2011), chronic non-communicable diseases are the leading cause of mortality in the world, representing $63 \%$ of all deaths. This percentage is even higher in Europe, in which these diseases suppose an estimated $86 \%$ of the deaths and a $77 \%$ of the disease burden in the region (WHO-EU, 2011). For this reason, it is important to develop strategies to try to prevent the onset of these diseases or to deal with its effects and keep the disease under control.

The Active Ageing, understood as the process of optimizing opportunities for health, participation and security in order to enhance quality of life as people age, is part of the process of coping with these chronic diseases, to try to maintain the elderly healthy and involved in the social live.

The REмоте project aims to help with the Active Ageing from a multidisciplinary and integrated approach to utilize information and communication technologies for addressing real needs of elderly people. The system not only will help the elderly to prevent or keep under control their chronic diseases, but also to exercise their mental health, by using Іст. The project will also benefit the professionals offering them ways to prescribe treatments (medical treatments, nutritional plans, etc.) for their patients and be able to do a follow up of the treatment. For the informal carers, REMOTE offers social tools, so they can also follow up their elderly health. Finally, the project also benefits the industry of health control products, which can be integrated within the services to be able to follow up the treatments.

In order to do so, REMOTE offers a set of services related to four different areas.

The first group of services is related to the environmental control at home. Those services control several sensors distributed in the elderly house. These sensors aim to convert the elderly house in a smart house, allowing him to control the lights, blinds, electric heating, etc. from their mobiles. There is also a system which controls the values of the sensors and warns the informal carers or the responsible authorities in case of an emergency.

The second group aims at helping the elderly to follow an organized schedule. This service is presented to the elderly as a calendar, where they can follow medical appointments, medication schedule, activity schedule, and any other reminder considered 
by their doctors or their informal carers or relatives. This is a basic service for the elderly with chronic diseases that live to their own, since the elderly usually tend to forget things, like taking their medication, which is basic to control their diseases and thus enable them to live longer and with a better quality of life.

Elderly people usually experience also some mental issues, like Alzheimer's. Therefore, the third group of services is related to help elderly to maintain their mental health through social gaming. This way, they exercise their minds at the same time they see it as

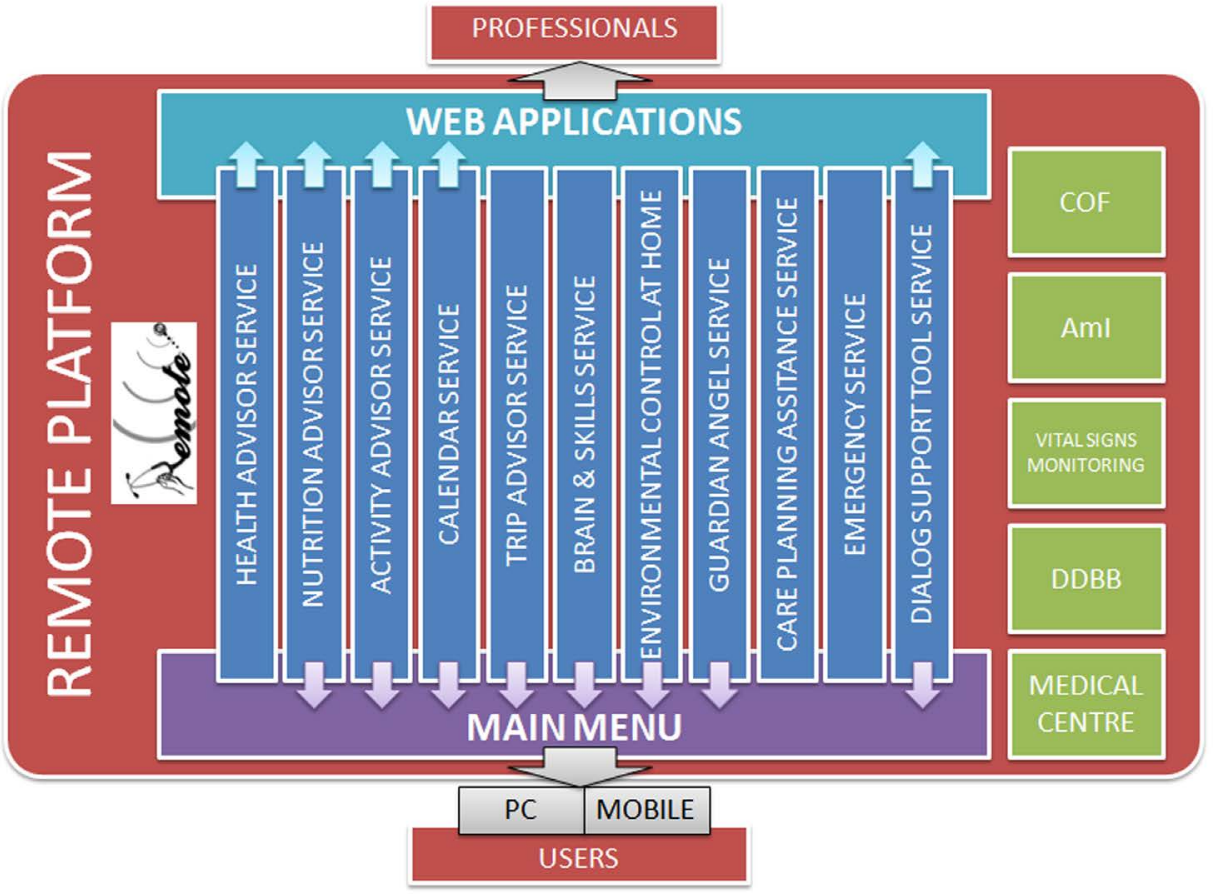
a game.

Finally, in this paper, we will deeply describe the fourth group of services, related to the physical health of the elderly. Elderly people with chronic diseases usually need to follow a strict diet, in order to control their diseases; they also take some medications that need to be controlled and, sometimes, reminded; also, in most cases, it is good for them to follow a simple exercise plan, to try to keep them fit and avoid movement problems or even paralysis that would make them dependent. To control these three areas, REMOTE offers the elderly a nutritional advisor, a medication advisor and an activity advisor. These services will help the doctors to define a concrete health program and the elderly to follow it.

Nowadays, the pilot phase of REMOTE has just started. The project will be tested in different countries, such as Norway, Spain, Israel, Romania, Germany and Greece. In each pilot, there are user interface (UI) specialists that test the applications before starting the user's test. When starting the user's test phase, the elderly participants, the doctors and the informal carers will be able to use the applications and prove the benefits of REMOTE. In this paper we will analyze the results obtained so far in the piloting.

Figure 1. REMOTE platform

This paper is structured as follows: section 2 describes the REMOTE platform; section 3 describes the physical health services; section 4 describes the piloting phase and finally section 5 exposes the conclusions of the work.

\section{The REMOTE platform}

The main goal of the REMOTE project is to offer the elderly a set of services that will help them in their daily lives. A person using REMOTE does not have to be subscribed to every service, but some.

REMOTE has implemented a platform in order to facilitate different server providers to provide services to the REMOTE users in a way that any other entity can create a client consuming those services, always integrated in a main menu.

Since REMOTE aims to offer a platform for e-health and social services, it is necessary a way to manage all provided services. In order to manage all services, the REMоте project has designed the Common Ontology Framework (COF), which provides a semantic-aware infrastructure that facilitates the discovery and invocation of web services. When a service provider offers a service, it needs to be integrated in the cof. This way we make sure all services are offered the same way, so an inde- 


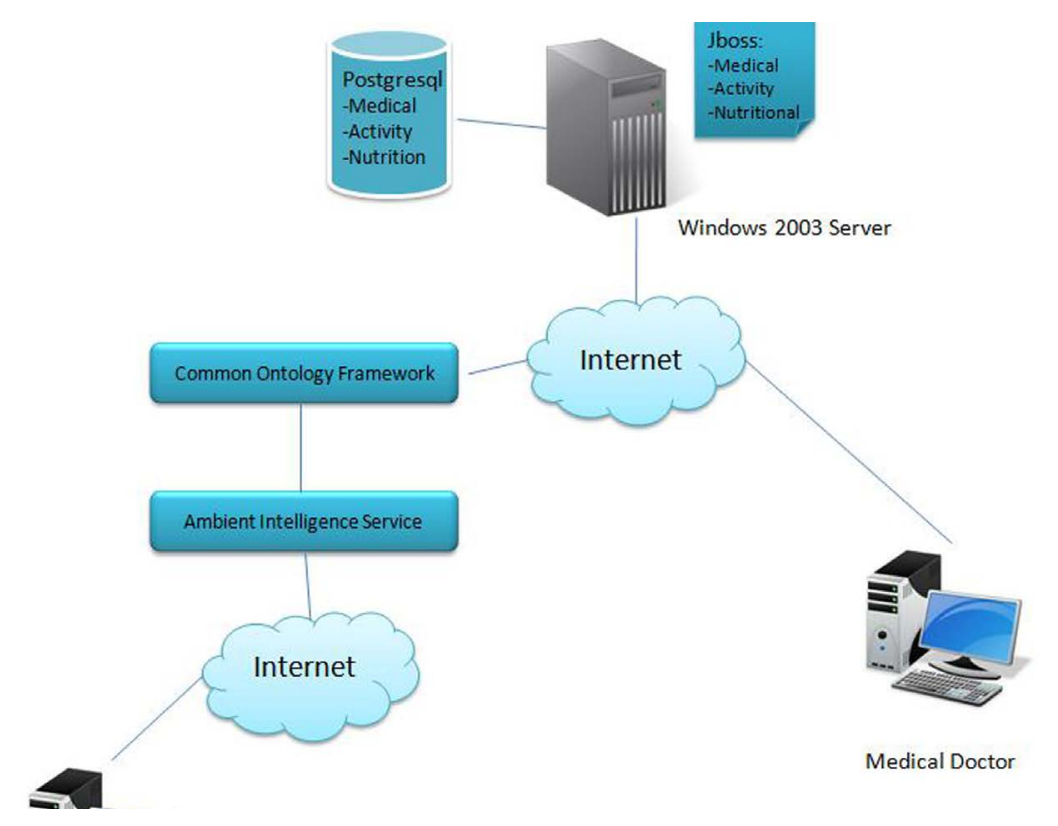

Figure 2. Services architecture

\section{The physical health related services architecture}

The goal of this paper is to describe the three services related to the physical health of the REMOTE users, the medical advisor, the nutritional advisor and the activity advisor.

All three services share a server-client based architecture, which can be seen in Figure 2 .

In all three cases, the server side runs in a Windows $2003^{\mathrm{Tm}}$ server, all three services are deployed in the same instance of jboss application server. pendent entity who wants to create a client for a service does not need to know how the service is implemented.

Then, when an entity wants to create a client that consumes that service, it must use the AmI framework which helps to discover REMOTE services and to implement the methods to connect with them. These services have all the same structure, so once someone has created a client for a service, it is easy to create a client for another one since the discovery and calls are executed the same way. Also, the client must be developed as an osGi bundle able to run in the main menu.

The main menu is a bundle specially developed to allow the elderly to have different REMOTE services running in the same environment, without the need of starting different applications. In the way it is designed, when starting the main menu it connects to the REMOTE service to check the credentials of the user and to download its profile. That profile grants the user access to the services he is subscribed and it is also used to automatically adapt the interfaces to the user needs, for example changing the colour combinations or the resolution of the main menu in case the user has any visual problems.

Figure 1 shows the complete REMOTE platform, with the services that are currently implemented.
Also, there is a PostgresQL ${ }^{\mathrm{TM}}$, DBMS, containing three data bases, one for each service, which contain all the information regarding the users that use that service, the doctors, and the specific data of each service, such as menus in the nutritional advisor case, activity plans in the activity advisor or medical plans in the medical advisor.

Once those services are deployed, in order to make them accessible to all the entities involved in the REMOTE project, they are integrated in the COF.

The user application is an osGi bundle running in Knopflerfish platform. This bundle has been developed to be integrated with the REMOTE main menu bundle. When the user starts the main menu, it searches for all the application bundles in the environment and present them to the user, so he can choose which application wants to run. The applications access to the profile which has been downloaded by the main menu to get the credentials of the users and access the service through the Ami using those credentials.

In the doctor's side, the doctor has a web application which is connected directly to the main server.

The services described in this paper do not have an application for the informal carers, but in the case 
it was, it will be another web application which will access directly to the service.

\section{Medical Advisor Service}

\subsection{The problem}

There are a lot of people that need medications in order to control a chronic disease or because they are eventually sick and they need prescription drugs to recover from that illness. One of the factors that can lead to treatment failure is mishandling it. It is really important to remember that, when you're following a treatment, the physician's instructions must be followed exactly and not dose should be missed.

There are different causes that can lead to mishandling the treatment or even to drop it, and these causes are usually more prevalent in older people. Some of them are:

- Neglect. Sometimes people do not even know what disease the drugs are treating or which benefits they are going to obtain from taking them. This leads to abandon the treatment, most times because they think those pills are not important.

- Some drugs carry side effects that can be nasty, like nausea, vomiting, headache, etc. and people think it is not worth it, so they drop the treatment.

- Sometimes we hear negative comments about a drug, on the media, from a relative, etc., and we prefer to listen to them instead of the doctor.

- In other occasions, people have difficulties to swallow the drugs, because they can swallow pills or they do not like the taste.

- It is also possible that you do not understand how to administrate the treatment, which leads to a mishandling of it.

- Another possible reason is that people do not know when they have to stop the treatment.

- And one of the most common causes of mishandled treatments is small slips, or confusions on the time the pills should be taken.
The mishandling of a treatment makes it ineffective, so it is important to help the elderly to manage their treatments.

A good administration of the medications will have a positive repercussion in the elderly's health, increasing their health status and improving their well being. This way, their life expectancy, as well as their independence, is increased.

\subsection{Medical assessment}

In order to set correctly the medical advisor, is extremely important to elaborate correctly the medical profile of the user.

There are several areas which need to be studied:

- Patient's health status: define the general health status of the patient.

- Patient's chronic diseases: studying the chronic diseases that the patient suffers has a major importance during the medical profiling. It is indispensable to know the diseases to set the medication.

- Medications the patient is taking: all medications that the patient is taking need to be characterized with its purpose, its main active, side effects and possible interaction with other medications.

Based on the information on the medical profile, the physician will elaborate a medical plan including all the medications that the patient must take and the complete instructions to follow the treatment correctly, as well as any interactions or any side effects the medicines can have.

\subsection{State of the art}

There are several projects working in similar applications to help the elderly to follow their treatments.

- OAsis: The oAsis project introduces a medication manager to follow up the user's medication schedule. It triggers the user to take his medication, informs him about any possible change (decided by the doctor), and checks that the user is following the schedule.

- Soprano: The soprano project has a medication alert which collects events from a medi- 
cation dispenser about missed medication taking, selects the most appropriate communication channel and delivers the alert to the assisted person (AP). In case the AP does not acknowledge the alert, the process is escalated and a sMs is sent to an informal caregiver.

- UniversaAL: The service provides a way to promote handling of medication to the assisted person. The system is able to check on available medication, to push proper alerts about medication intake times and to acknowledge that the proper medication has been taken.

\subsection{The REMOTE's solution}

Remote medical advisor shows the elderly their medication plans in the calendar and triggers alerts when a medicament has to be taken. When an intake alert is triggered, the user can check that he has taken the medication. This will allow the doctors to do a follow up of the treatment from their web application.

Also, REMOTE offers a mobile application. This way, the patients will have the alerts even when they are outside the house. This application aims to promote the users to leave the house and not be always aware of the desktop application, allowing them to have an active social live, which is essential for the elderly mental health.

\subsection{Doctors' application features}

The doctors' web-based application allows them to consult a patient's medication plan, assign a new medication to a patient, and introduce new medicaments in the data base.

Other important feature is that the doctors can consult when the patients have marked the medication as taken. It also allows doctors to fill the patient's health record and to consult it.

There are other features in the web page related to other REMOTE services, such as the alerts services that registers alerts from the environmental control, or the vital signs service, which register the vital signs of the patients.
The final user is able to see his medication schedule in a calendar application, specially developed to be integrated in the REмоте's main menu. This calendar shows the user not only the medications, but also appointments with the doctor, or their families, their menu for the day, or any other remainder set by the doctor or the informal carer.

Regarding the medication, the calendar shows a pop-up window when the user has to take a medication, with information about the medicament and the dose. Then, the user has a button to assert that he has taken the medications, so the doctor can control that the treatment is being followed correctly.

\subsection{Expected benefits}

The main benefit expected from this service is to improve the way the elderly follows the medical treatments. The fact that the calendar shows popup alerts when a medication must be taken will prevent that the elderly forgets to take it.

Also, another benefit is that the doctor is able to control how the patient is following the treatment. This feature allows that, in case of failure of the treatment, the doctor could study if it has happened because it was not the appropriate treatment or because it has not been followed properly.

It is also expected a cost reduction for the health provider entity due to the better control of the patient and the higher utilization of doctors' time, as they can review the patient's status at time slots when they are not visited by patients.

\section{Nutritional Advisor Service}

\subsection{The problem}

As Hippocrates said in $400 \mathrm{BC}$, "let food be your medicine and medicine be your food".

The food that we eat plays a very important role in our health. A balanced diet comprising of diverse and healthy foods is key to promoting good health. It is also proved that food can be harmful and cause or worse several diseases (A Cruz, Hales).

\subsection{User's application features}


For those reasons, we need to take care of our diet, and to take especially care of the elderly diet, so they can increase the quality of their life.

In developed societies, elderly population has a higher risk of undernourishment than young people, due to different pathological and physiological factors (Fukui, Phillips, Roberts).

Inadequate food intake, dietary deficiencies due to incorrect food choices and diseases that can cause increased nutrient requirements, increased nutrient loss or poor nutrient absorption can lead to malnutrition. So it is needed to identify the factors that can lead an elderly to undernourishment in order to define how to intervene effectively.

Chronic diseases also have a nutritional component. For instance, obesity is a major risk factor for cardiovascular diseases and it also aggravates chronic obstructive pulmonary diseases. Alcohol increases the risk of stroke linearly, and fat intakes have been related to cerebral infarct. Several factors are involved in the development of some cancers, like tobacco, diet, alcohol, infections and hormones. Type II diabetes is related to body fat and its distribution in the abdominal area.

Since several chronic diseases are closely related to nutritional habits, defining prevention nutrition strategies would improve the elderly's life, by preventing or decreasing the risk of those diseases.

There are several phases in the prevention through nutrition (WHO, 2002), depending on the stage of the disease:

- Primary prevention involves risk-factor modification to prevent the occurrence of disease.

- Secondary prevention involves screening for a disease before it becomes symptomatic.

- Tertiary prevention involves treating and minimizing the complications of a disease once it is developed.

The rising prevalence of chronic diseases in ageing populations is a substantial burden not only for health care systems but also for social and family structures due to dependency resulting from the elderly status. So health systems need to take a new approach focused on proper nutritional habits and healthy nutrition promotion that enhances the well-being of elderly users and decreases the chronic diseases incidence.

The nutritional plans elaborated by nutritionists are often abandoned by the elderly due to several factors as for instance they do not like the meals in the menus or the lack of motivation, or because they do not have the feeling that the diet is working. So a system is needed which allows the nutritionist to take into account these factors and motivates the elderly to continue with their nutritional plan.

\subsection{Medical assessment}

The main purpose of nutritional profiling is to detect nutritional risks and preferences of the users, so the nutritionist can determine the nutritional plan.

The main areas which need to be studied are:

- Diet history, which refers to the usual pattern of food intake and the food selection variables that dictate food intake.

- Anthropometric data, which refers to the study of the measurement of the human body in terms of the dimensions of bone, muscle, and adipose (fat) tissue.

- Clinical history and biochemical parameters, which refers to data gathered by the practitioner about the patient's health with the aim of obtaining information for formulating a diagnosis and providing support to the patient.

- Physical activity evaluation, which refers to the physical activity that the elderly practices.

Once all this information is gathered, the nutritionist can use it to fill the nutritional report of the patient, which includes current weight, body mass index, fat percentage, total energy expenditure, total energy intake, likes, dislikes, allergies and intolerances, relevant health issues, medicine interactions and the nutritional goals.

With the information of the nutritional report, the nutritionist must elaborate a nutrition plan for the user. This plan is not only the daily menus, but also should provide patients with qualitative recommendations about nutrition tailored to their needs, in order to re-educate their nutritional habits. 


\subsection{State of the art}

Currently, there are three European projects dealing with the creation of systems to improve elderly nutrition.

- PIPs: The objective is to encompass the entire set of business processes, professional practices, and products applied to the analysis and preservation of the citizen's well-being using the latest innovations in ICT. Among other services, PIPs offers a Personalized Nutritional Support which offers: personalized nutritional advisor, shopping list, product information, product check according to personal profile, etc. The aim of this service is to provide its users with information and advice about having a healthy lifestyle and choosing healthy nutritional habits that are tailored to the user's needs.

- Persona: persona aims at advancing the paradigm of Ambient Intelligence through the harmonization of Ambient Assisted Living (AAL) technologies and concepts for the development of sustainable and affordable solutions for the social inclusion and independent living of senior citizens, integrated in a common semantic framework. The Persona project has several services related to nutrition as a nutritional advisor, a cooking assistant, a shopping assistant and a food inventory.

- OAsis: OAsis introduces an innovative, ontology-driven, open reference architecture and Platform, which will enable and facilitate interoperability, seamless connectivity and sharing of content between different services and ontologies in all application domains relevant to applications for the elderly and beyond. The OASIs project includes a nutritional advisor which proposes a weekly based menu, helps with the shopping list, offers tips and advices and offers the elderly several questionnaires which can help the nutritionist to better adjust the diet.

\subsection{The REMOTE's solution}

The nutritional advisor of the REMOTE project has been inspired by the nutritional advisor from the
OASIS project, since both projects share aims, with different target users. In the case of REMOTE, target users are elderly people with several problems, so the application has been simplified in order to facilitate this usage. Also, the web application for the doctors is new in REMOTE, so they can easily assign nutritional menus.

The patients also have mobile applications ready for them, so they do not need to be at home to follow the nutritional plan. For example, if they are visiting their relatives, they can let them know what they are supposed to eat, so they do not skip the plan.

\subsection{Doctors' application features}

The nutritional advisor offers doctors a web-based application which allows them to:

- Assign weekly menus to a patient.

- Adjust the calories of a menu.

- Check if the patient is following the menu and if he likes it.

- Create new weekly menus.

- Create new meals for the menus.

- Create new recipes.

- Introduce new ingredients in the data base.

- Create new advises for a patient.

All this features allow doctors to personalize the menus for their patients, in order to create the most appropriate menu for them. It also allows them to control if the patient is following the nutritional plan; or in case he is not following it, if it is because he does not like the meals. Also, doctors can add personalized advises to their patients, to encourage them to follow the diet.

\subsection{User's application features}

The nutritional advisor is the most complete application in REMOTE. It allows users to:

- Consult the menu for today and tomorrow.

- Change a meal scheduled for today for the one scheduled for tomorrow.

- Inform the nutritionist about if they had eaten the meals and if they liked them.

- Check the menu for all week. 
- See the recipes of a meal.

- Check for advises related to nutrition.

- Elaborate a personalized shopping list, from one day to all week long, based on their nutritional plan.

- Add items to the list outside the basic ones to elaborate their menu.

- Print the shopping list or send it to a carer person who can go shopping for them.

All this features facilitate the elderly to keep on with their diets.

\subsection{Expected benefits}

Nutrition is a very important aspect of keeping a healthy life, and it is even more important for the elderly, who usually suffer from diseases related to bad nutritional habits, such as diabetes, cholesterol, high blood pressure, etc.

The main benefit expected from this service is to help users to maintain a diet provided by their nutritionists. If the elderly have a service that tells them what they should be eating for each meal every day, and that helps them to elaborate a shopping list according to their diets, it would be easier that they don't drop out the plan.

The nutritional advisor is also integrated with the calendar application, so an alert is shown in the eating hours defined by the patient, which will also help them to follow the diet.

It is also expected a long term cost reduction for the health provider entity, since a good nutrition will keep the elderly healthy, avoiding or delaying chronic diseases that suppose a cost in treatments, hospitalizations and carers.

\section{Activity Advisor Service}

\subsection{The problem}

Physical activity can play an important role in increasing the independent living of the elderly. Maintaining a good physical health helps elderly people to maximize life expectancy and to achieve a better quality of life.
Keeping fit can help old people to increase their levels of energy and to have a general sense of wellbeing. It also helps prevent or mitigate the effects of several diseases like blood pressure, diabetes, lipid profile, osteoarthritis, osteoporosis, and neuro-cognitive function. It has also been proved that regular activity reduces mortality and age-related morbidity in the elderly (Med Sci).

Most of our elderly can be classified as sedentary. They were not used to practice exercise when they were younger so they have not started to practice it as old, or maybe, even if they were used to practice some sport, they stopped years ago.

That lack of physical activity can bring our elderly to suffer unnecessary illness or aggravate existing ones, which leads to lose their independence.

In order to increase the life expectancy and to extend as much as possible the independence of the elderly, it is important that they follow an exercise plan which should be designed according to their needs.

Based on the diseases they suffer, or the ones they are going to probably develop, the medications they are taking and the diet they follow, a professional can design an activity plan which helps the elderly to keep fit and to maintain their diseases as controlled as possible.

It is important that the plan fits with the possibilities of the elderly. For instance, you cannot design an exercise plan that includes static bike if they have problems in their knees.

\subsection{Medical assessment}

In order to define the activity plan that better suits with the user's needs, it is important to elaborate a profile of the user. The aspects that should be considered are:

- User's health status: define the general health status of the patient.

- User's physical problems: it is important to know if the user has any problem that prevents him from practicing any kind of exercises.

- User's activity history: talk to the user and get to know how used he is to practice some exer- 


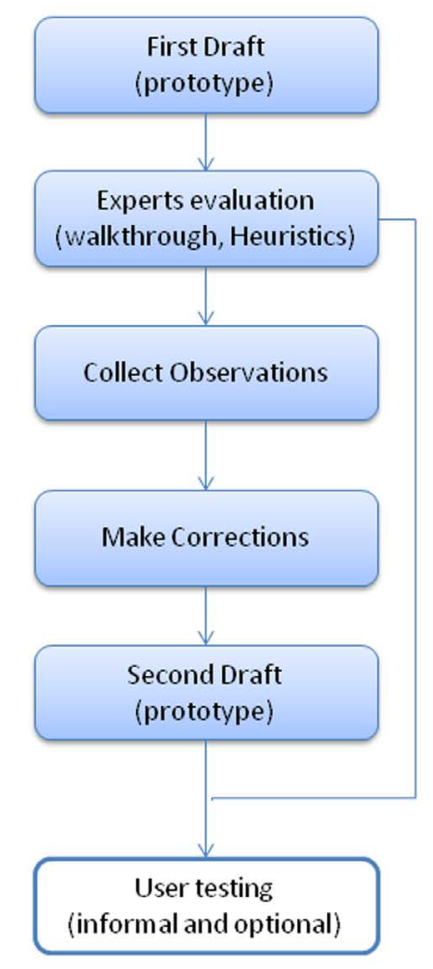

Figure 3. Phase one of evaluation plan. Expert's evaluation

cise, what kind of physical activity he practices or he would feel comfortable practicing, etc.

This information will be stored in the user profile and will help the professional to elaborate an activity plan according to the user's needs and preferences, so he can feel comfortable following it. The main aim of the plan should be to keep the user fit and to increase his well being and life expectancy.

The assessment will provide the activity advisor with the necessary information to present the user the activity plan and help him follow it.

\subsection{State of the art}

There are several projects that are working in some kind of applications to help the elderly to follow an activity plan.

- Persona: In this project an outdoor activity monitor monitors the level of activity when the user is outside home moving around the neighbourhood or town. It uses that informa-

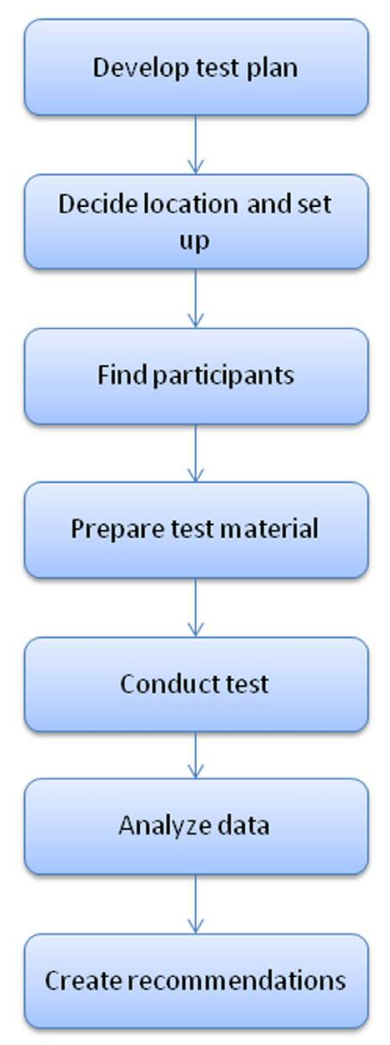

Figure 4. Phase two of evaluation plan. User's evaluation

tion to motivate the user to leave home and to walk around with friends or goi shopping. At the end of the day, the final aim is to orient the user to follow a healthy lifestyle through the motivation of physical activities.

- OAsis: The OAsIs project introduces the activity coach, which aims not only to present an activity plan, but also to control it through sensors, recognize the activity that its being done and characterize the physical activity through the calculation of parameters such as speed and running/walking distance, as well as time spent in exercising.

\subsection{The REMOTE's solution}

The REMOTE project facilitates the user to do the exercise plans by offering them an easy to follow interface, with all the information needed to perform the exercise. Also, the users may have a mobile application, which is a copy of the desktop one, 
so they can consult and perform their activities when they are outside their home.

Also, their patients will perform the activity with a vital signs belt, so they can be monitored all time by the doctors.

\subsection{Doctors' application features}

The web-based application designed for doctors allows them to introduce new exercises in the data base and to create a personalized activity plan for their patients. They can choose the number of phases of the plan and the number of exercises inside each phase.

They can also add new exercises to the data base, in case the appropriate one for their patients is not in the data base.

\subsection{User's application features}

The activity advisor PC desktop application offers users the possibility to see their complete activity plan, to personalize the days they want to do the exercises of current phase and to check the exercises they have scheduled for today.

Users do not directly mark exercises as done, as they do in the nutritional advisor. They are supposed to perform their exercises wearing a sensewear device which catches their vital signs and send them to another REMOTE service, the vital signs monitoring.

\subsection{Expected benefits}

In order to be independent for as long as possible, it is very important that the elderly are fit. If they stop moving and they do not practice a little exercise, their muscles can atrophy and they may lose mobility, which will cause a dependency.

The main benefit expected from this service is to help keep elderly people fit and in movement for as long as possible. This way, we increase the probability that they live a longer healthy and independent life.

Avoiding dependency will also save money to the health provider entities, since the elderly will not need a carer dedicated to them.

\section{Piloting}

The evaluation plan for the REMOTE project has been designed as a two phase plan, with one phase where the applications will be evaluated by experts and a second phase where the applications will be tested by users.

\subsection{Phase 1: Expert-based evaluation}

The main goal of the expert-based evaluation is to discover potential violations to accessibility and usability standards in the design before the applications reach production. In order to do so, the evaluation will follow the typical iteractive design process format which involves the steady improvement of the design based on user testing.

Figure 3 shows the diagram of the iteractive design process, whose steps are:

- First draft: the developers will deliver an initial prototype of the application.

- Expert's evaluation: several user-interface experts will walkthrough to note any potential problematic areas and a heuristic analysis.

- Collect observations: collect the observations from the walkthroughs and the heuristic analysis from each expert and discuss the findings and ways to correct the problems noted.

- Make corrections: correct the initial design with the observations of the experts.

- Second draft: repeat steps 2-4 until the user interface problems noted by the experts are resolved.

- User's testing: this step is optional and involves users doing a test of the application before the official phase.

\subsection{Phase 2: User-based evaluation}

The second phase of REMOTE's proposed evaluation program involves user-based evaluation. User-based evaluation can be done at any stage of the product development lifestyle, but it is preferable to start when a working prototype of the application is in place.

Usability testing is the most fundamental userbased evaluation method because it provides di- 
rect input on how the user perceives and interacts with the application or interface that is being tested and on whether the design areas create confusion and other problems to the user. The value of observing a real user trying to go through real case scenarios is truly irreplaceable as the team can have a clear view of the design's shortcomings and successes.

Figure 4 shows the diagram of the usability testing process, whose steps are:

- Develop test plan. During this phase it is necessary to define three things: what is going to be tested; why is the test being conducted; and how is the test going to be.

- Decide location and set up. Decide on the location where the test will be conducted i.e., lab, office, remotely, or locally, as well as the equipment that is going to be needed i.e. cameras, software, hardware etc. REMOTE tests can be conducted, for example, in context of purposefully organized demo days and/or during the running of the pilots.

- Find participants. Select the users that are going to participate in the test. Define the profile of the real user of the system that is being tested and then select testers that fit that profile as close as possible.

- Prepare test material. Have printed copies of all the documents that are going to be used during the test.

- Conduct test. There has to be an introduction to the users of what they have to do and leave them time to perform the testing.

- Analyze data. The usability specialists will go over the observations and the comments that were made by the users during the test. The data acquired from the think aloud method will be reviewed by the usability specialists and translated into concrete usability and accessibility concerns.

- Create recommendations. Based on the data gathered during the test, the usability specialists will create a list of recommendations on what needs to be changed in order to make the system more user-friendly and accessible and solutions to problems that were observed. The results will be then shared and discussed with the rest of the development team.

\subsection{Current stage on REMOTE evaluation phase}

REMоте started its evaluation phase on October 2011. Nowadays, the expert's evaluations phase of the three services presented in this paper is being conducted in Germany, Romania, Greece, Israel, Spain and Norway.

The evaluation is being conducted over an advanced working prototype of the complete systems. Several UI experts in each country are testing the doctors and users applications in order to check if they offer all the necessary functionalities and if they do not have any violations to the accessibility.

So far, experts have found small problems regarding to doctor's usability of the health record and the way to elaborate menus in the nutritional advisor.

The health record of the patients is an important part of the medical advisor, since doctors need to know it in order to prescribe the correct treatments. The UI experts have expressed concerns about the order of the different form fields and the way doctors store the records. We are currently working on better distribution of the form fields and an easier way to store and retrieve the information.

Regarding the nutritional advisor, the experts concerns were about the difficulty of creating weekly menus. A weekly menu is a much elaborated object, which contains at least three meals per day, which are compound by different recipes, at the same time compound by ingredients. All these amount of data is overwhelming and difficult to show in a single screen, so currently there are several tabs with the ingredients, the recipes and the meals, and finally a tab to create a weekly menu by selecting meals. Nowadays, we are working on a better distribution of the information to solve this problem.

Regarding the phase 2 of the evaluation, the first steps have already been executed. There is a group of 150 volunteers in six different countries (Spain, Norway, Germany, Israel, Romania and Greece) 
which will perform the testing. Each piloting partner has defined his own evaluation scenario, where different REMOTE services will be tested with different users.

The phase 2 of the evaluation is set to start in May 2012, so at this point we do not have any feedback from end users yet.

\section{Conclusions and future work}

This paper presents a group of REMOTE services which aim to help elderly people with their physical health. It is expected that these services contribute to maintain the elderly healthy and to help them to live independently for a longer time.

During the project, several applications have been developed for the elderly, the informal carers and the professionals, so they can use the REMOTE services. These applications have been designed following the lines drawn by experts in applications for the elderly, which are participating in the piloting. Those experts have been involved in all development phases, guiding the developers.

Also, the interfaces have followed the principles of accessibility and adaptability, since the elderly participants in the project have different conditions which require different interfaces. An automatic adaptation system has been developed, which adapts the interface of the application to the visual conditions of the user.

The three services presented in this paper aim at helping the elderly to follow their medical treatments, their nutritional plans and an exercise pro- gram. Combining these three actions will help the elderly to keep fit, and therefore the possibility of living independently for longer.

Apart from the expected benefits for the elderly, we also aim to prove that these services can help the health service providers to save money by three means:

- They will reduce spending on carers if the elderly are able to live on their own.

- If doctors have a system to easily assign plans to patients and control if they are following them that would optimize doctors' work by saving time.

- There is also a cost reduction in the long run due to better control over the patient and minimizing hospitalization time.

Since the evaluation phase has recently started, there are still no results that can confirm the expected benefits, although is expect to be able to confirm them in a short term.

The REMOTE project is approaching its final stage, starting the evaluation phase. The future plans include improving the applications to adapt them better to the users' needs and to analyze the results of the evaluation, so we can prove if the applications are useful.

\section{Acknowledgement}

The REMOTE project is financed by the European Union, inside the Ambient Assisted Living Joint Programme (AAL Call 2008-01). 


\section{References}

Cruz, A. M.L., Wong, W. W., Mimouni, F., Hachey, D. L., Setchell, K.D.R., Kelin, D. P. and Tsang, C.T. Effects of Infant Nutrition on Cholesterol Synthesis Rates. Pediatric Research (1994).

EU (2009). Europe's response to world ageing. http://europa.eu/legislation_summaries/employment_and_social_policy/disability_and_old_age/em0020_en.htm

Fukui K, O. K., Shinkai, T., Suzuki, S. and Urano, S. Impairment of learning and memory in rats caused by oxidative stress and aging, and changes in antioxidative defense systems. Ann N Y Acad Sci, 2001. 928(168): 75.

Hales, C. N. and Barker, D. J. P. , Type 2 (non-insulin-dependent) diabetes mellitus: the thrifty phenotype hypothesis. Diabetologia, Volume 35, Number 7, 595-601.

Isaacs, B. (1972). Survival of the Unfittest. Routledge and Kegan Paul Ldt. Brodway House.

Med Sci Sports Exerc, American College of Sports Medicine Position Stand. Exercise and physical activity for older adults. 1998; 30:992-1008.

OASIS. Open architecture for Accessible Services Integration and Standardization. http://www.oasis-project.eu PERSONA IP-EU-project (FP6). 20th of January, 2010. Available from: http://www.aal-persona.org

Phillips PA, R.B., and Ledingham J.G., Reduced thirst after water deprivation in healthy elderly men. N Engl J Med 1984. 311(753-9).

PIPS, Hon Project. Available from: http://www.hon.ch/Project/HONProjectsPIPS.html.

Roberts, S. B. and Regulation of energy intake in relation to metabolic state and nutritional status. European Journal Clin Nutrition, 2000. 54(S64-9).

SOPRANO. Service-oriented Programable Smart Environments for Older Europeans. http://www.soprano-ip.org/

UniversAAL. univerAAL open platform and reference Specification for Ambient Assisted Living. http://www.universaal.org/

WHO (2002). Keep fit for life. Meeting the nutritional needs of older persons. Geneva.

WHO (2011), World Health Organization, Chronic diseases. http://www.who.int/topics/chronic_diseases/en/

WHO-EU (2011). Non-communicable diseases in Europe Region. http://www.euro.who.int/en/what-we-do/healthtopics/noncommunicable-diseases 